\title{
Recombination Reactions of Atomic Chlorine in Compressed Gases. 1. Chemiluminescence Spectra of Chlorine Molecules with Argon Pressure Up to 170 bar
}

\author{
L.-C. Chang, T.-T. Song, C.-C. Tai, and T.-M. Su* \\ Department of Chemistry, National Taiwan University, Taipei, Taiwan, R.O.C., and The Institute of the Atomic \\ and Molecular Sciences, Academia Sinica, Taipei, Taiwan, R.O.C.
}

Received: February 19, 1996; In Final Form: April 23, $1996^{\otimes}$

\begin{abstract}
The gas-phase chemiluminescence spectra of chlorine atom recombination reactions in argon with pressures up to 170 bar were recorded by the laser photolysis/chemiluminescence detection scheme. With the spectroscopic data available in the literature and the monochromator functions determined experimentally and by assuming that the pressure line broadenings and frequency shifts follow the conventional Lorentzian form, for the first time the chemiluminescence spectra were quantitatively decomposed into the emissions of the $B^{3} \Pi\left(0_{\mathrm{u}}{ }^{+}\right) \rightarrow X^{1} \Sigma_{\mathrm{g}}{ }^{+}$and $A^{3} \Pi\left(1_{\mathrm{u}}\right) \rightarrow X^{1} \Sigma_{\mathrm{g}}{ }^{+}$transitions. The relative luminescence weights of these two emission electronic states, the vibrational temperatures, the pressure line broadenings, and frequency shifts were obtained. Their implications on the mechanisms of the chlorine atom recombination reactions and the interaction strength between the chlorine molecule and argon atom were discussed.
\end{abstract}

\section{Introduction}

The gas-phase chemiluminescence spectra of chlorine atom recombination reactions have been studied by several researchers since the 1960s. ${ }^{1-7}$ It has been well-established that the chemiluminescence spectrum is mainly due to the $B{ }^{3} \Pi\left(0_{\mathrm{u}}{ }^{+}\right)$ $\rightarrow X^{1} \Sigma_{\mathrm{g}}{ }^{+}$transition. Although there was evidence which suggested that the $A{ }^{3} \Pi\left(1_{\mathrm{u}}\right) \rightarrow X^{1} \Sigma_{\mathrm{g}}{ }^{+}$system could be embedded in the main emission in the chlorine atom recombination reaction and also in the nitrogen trichloride decomposition flame, ${ }^{6,7}$ these emission bands were usually assumed to be comparatively weak and therefore ignored in the traditional analysis of the recombination emission spectra. ${ }^{8-10}$ The unavailability of reliable spectroscopic data for the $A$ state at that time prevented a good analysis of the $A \rightarrow X$ emission bands in the gas-phase atomic chlorine recombination reaction. In contrast to these gas-phase experiments, the emission of the chlorine molecules in a $4 \mathrm{~K}$ argon matrix was assigned as the $A^{\prime}{ }^{3} \Pi\left(2_{\mathrm{u}}\right) \rightarrow X^{1} \Sigma_{\mathrm{g}}{ }^{+}$transition. $^{11,12}$ No other types of emission were observed in these experiments.

It has been well recognized that the recombination emission spectra, under either stationary-state or transient conditions, carry the kinetics information of the recombining species. ${ }^{1-10,13-15}$ In this report, the laser photolysis/chemiluminescence detection method was employed to study the emission spectra of the atomic chlorine recombination up to an argon pressure of 170 bar. This low-to-medium pressure spectroscopic study is a necessary step in understanding the kinetics of the chlorine atom recombination up to medium pressure, especially for the demonstration of the behavior of the geminate recombination of the chlorine atoms in this pressure regime. The latter topic constitutes the second part of this series of atomic recombination study. ${ }^{16}$

In the present study, based on the recent precise spectroscopic data of the $A$ state obtained by Ishiwata et al., ${ }^{17}$ the chemiluminescence spectra of the chlorine atom recombination reaction in various argon pressures were analyzed and the quantitative population relation among the vibrational states of the $A$ and $B$ states were obtained. The pressure-dependent parameters, such

* To whom correspondence should be addressed at National Taiwan University.

${ }^{\otimes}$ Abstract published in Advance ACS Abstracts, July 1, 1996.

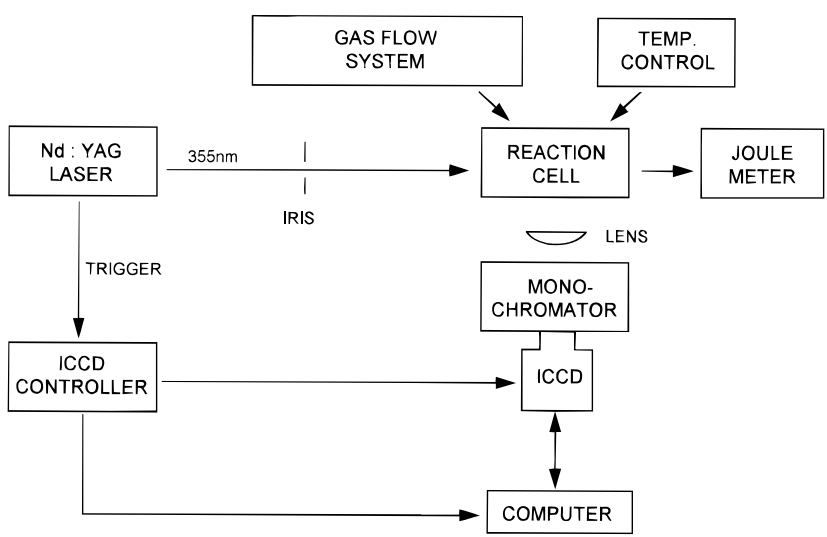

Figure 1. Schematic diagram of the experimental setup for the lowpressure experiments. In the high-pressure cases, the flow reactor was replaced by a high-pressure bomb.

as the pressure line broadening and the frequency shifts, were also determined in the present spectral intensity simulations. The traditional interpretation of the afterglow spectra of the chlorine atom recombination reaction is reassessed and its implications on the mechanism of the chlorine atom recombination reaction is discussed. The possible $A^{\prime} \rightarrow X$ emission as suggested by the matrix isolation experiments was also looked for under the present medium pressure conditions.

\section{Experimental Section}

Figure 1 shows the schematic block diagram of the experimental setup. The $355 \mathrm{~nm}$ laser pulse with a duration of $5 \mathrm{~ns}$ of a Nd:YAG laser was employed to dissociate the chlorine molecules. Electronic ground-state chlorine atoms were generated. For the experiments with total pressure below 1 bar, the main reaction chamber is a flow reactor constructed from a stainless steel cube of $15 \mathrm{~cm}$ for each side. To ensure the constancy of the sample composition, small flows of argon and/ or chlorine gases were introduced separately but coaxially into the reaction cell by two flow controllers. For experiments with total pressure being higher than 1 bar, a high-pressure reaction cell whose main body was composed of a cylindrical bomb with $9.5 \mathrm{~cm}$ inner diameter and $13 \mathrm{~cm}$ depth (Parr Instrument) was 
used. Four quartz windows, each with a diameter of $2.54 \mathrm{~cm}$, were attached to the cylinder wall in perpendicular positions with respect to each other. Since in the present high-pressure experiments there is no depletion of the chlorine atoms due to possible wall reactions, the gas samples were only regularly replaced after certain experimental runs. For most of these highpressure experiments, the chlorine pressure was kept at 5.33 mbar. The argon pressure was varied from 1 to 180 bar. The sample cell was kept at $300 \mathrm{~K}$ by a thermostat. The chlorine (natural isotope abundance; semiconductor grade, $99.997 \%$; Scott Specialty Gases) and argon (99.9995\%, Scott Specialty Gases) were used directly from the gas cylinders without further purification. The laser energy was monitored by a calibrated pyroelectric joule meter and kept at a level such that the chlorine atom concentration at the center of the reaction cell was around $2 \times 10^{14}$ molecules $/ \mathrm{cm}^{3}$.

The recombination emission was dispersed with a $275 \mathrm{~mm}$ focal length monochromator (Acton Research) with a reciprocal linear dispersion of $3 \mathrm{~nm} / \mathrm{mm}$. Depending on the pressure, the slit width was set at $0.070 \mathrm{~mm}$ for the low-pressure experiments and $0.120 \mathrm{~mm}$ for the medium-to-high pressure measurements. The corresponding measured monochromator-function widths (fwhm) fitted by the Gaussian form were 0.300 and $0.409 \mathrm{~nm}$, respectively. The photons were then detected by a gated intensified charge-coupled-device (ICCD, Princeton Instruments). The normal timing sequences of the experiments were usually a delay of a few microseconds after the laser shot and then followed by a gate opening varying from few hundred microseconds to $10 \mathrm{~ms}$ for the chemiluminescence signal. The exact time duration was determined by the chlorine atom recombination time as measured in the corresponding transient experiments. ${ }^{16}$ To enhance the $\mathrm{S} / \mathrm{N}$ noise ratio, around 50000 laser shots were accumulated for each spectrum. A standard spectral radiance source (Eppley Laboratory, Inc.) was employed to calibrate the detection efficiency of the present optical and electronic detection systems.

\section{Results and Discussion}

Figure 2 shows the potential energy curves of the $X, A^{\prime}, A$, $B^{\prime}, B$, and $C$ states of $\mathrm{Cl}_{2}$ near the dissociation energy. The $C$ state potential was that derived by Gibson et al. ${ }^{18}$ along with the extrapolation of the potential functional form $C_{8} r^{-8}$ to the dissociation limit. The remaining bound-state potentials are the reported RKR potentials along with the extrapolation of the potential functional form $-C_{6} r^{-6}-C_{8} r^{-8}-C_{10} r^{-10}$ to the dissociation limit. ${ }^{17,19-22}$ For these bound states, $C_{n}$ 's were determined by fitting the potentials to the highest three RKR potential points on the dissociation branch. The following spectral analysis and discussion shall be based on these potential energy curves.

Analysis of the Chemiluminescence Spectra in the LowPressure Regime. Figure 3 a shows the chemiluminescence spectrum obtained at neat chlorine gas of 4.0 mbar. The luminescence intensity has been corrected by the photon detection efficiency of the detection systems. The vacuum wavelengths were used in the figures. The general features of the spectrum are in agreement with the earlier results obtained by conventional flow-discharge methods. ${ }^{8}$ Since it has been well-established that the main emission is due to the $B \rightarrow X$ transition, the spectrum was first simulated solely with this transition in mind. With the reported RKR potentials of the $B$ and $X$ states, ${ }^{19,20}$ the Franck-Condon factors calculated by Cooley's routine, ${ }^{23}$ the Hönl-London factors for the rotational line strengths, the natural abundance of the chlorine molecules, and assuming that the rotational temperature was at the

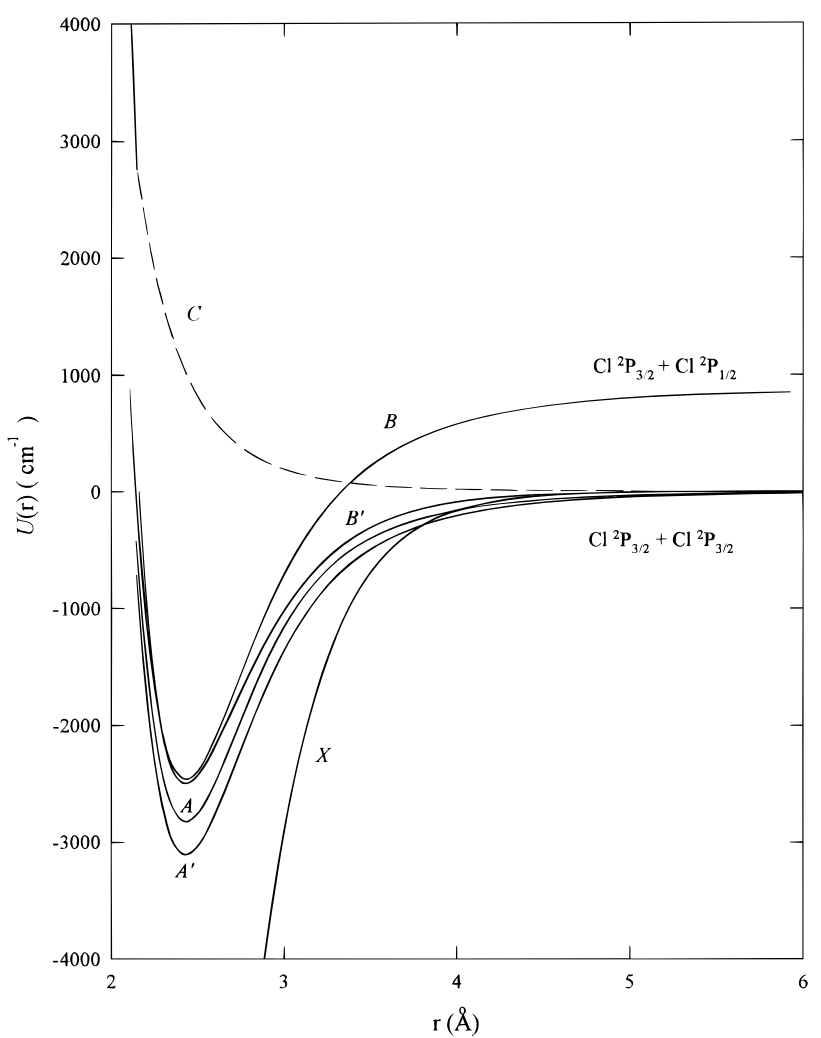

Figure 2. Potential energy curves of the $X, A^{\prime}, A, B^{\prime}, B$, and $C$ states of $\mathrm{Cl}_{2}$ near the dissociation limit. See the text for the details.

experimental temperature of $300 \mathrm{~K}$, a theoretical spectrum with adjustable vibrational populations was obtained. The monochromator function employed in the simulation was a Gaussian function obtained in the least-squares fitting to the measured mercury line spectrum. It was found that there were always substantial residual photon emission between the experimental and the simulated $B \rightarrow X$ emission bands, whose band structures exactly correspond to the $A \rightarrow X$ transition reported by Ishiwata et al. ${ }^{17}$ With the RKR potential of the $A$ state, the theoretical $A \rightarrow X$ emission spectrum could be obtained in a similar manner. By introducing an additional fitted parameter-the relative luminescence weights of the $A$ and $B$ states-the complete radiative recombination spectrum could be simulated quantitatively. For comparison, in the simulated spectra the total peak value of $B\left(v^{\prime}=0\right) \rightarrow X\left(v^{\prime \prime}=11\right)$ vibrational band at $842.6 \mathrm{~nm}$ was normalized to the corresponding experimental value.

Figure $3 \mathrm{~b}$ shows the total simulation spectrum, and Figure $3 \mathrm{c}$ shows the percentage deviations between the theoretical values and the experimental results over the wavelength range $650-893 \mathrm{~nm}$. The average percentage deviation is $6.5 \%$ and the maximum percentage deviation is about $\pm 10 \%$. The few random large-amplitude spikes of Figure 3c, which are usually larger than $\pm 10 \%$, are mainly due to the defects of some picture cells of the ICCD, which are more pronounced in the low-signal region, and also partly due to the small uncertainty of the wavelength in the scanning of the monochromator. As shown in the figure, the agreement between the experimental and simulated spectrum is excellent.

Figure 4a,b shows the contributions of the $A \rightarrow X$ and $B \rightarrow$ $X$ transitions to the total emission spectrum, respectively. The main vibrational bands of the two major isotopic species are also indicated in the figure. The fitted relative luminescence weight of the $A$ and $B$ states, which is the ratio of the product of the population and the square of the transition dipole moment of the $A$ state to that of the $B$ state, is 0.50 . The fitted vibrational populations of the $B$ state are basically in a Boltzmann 

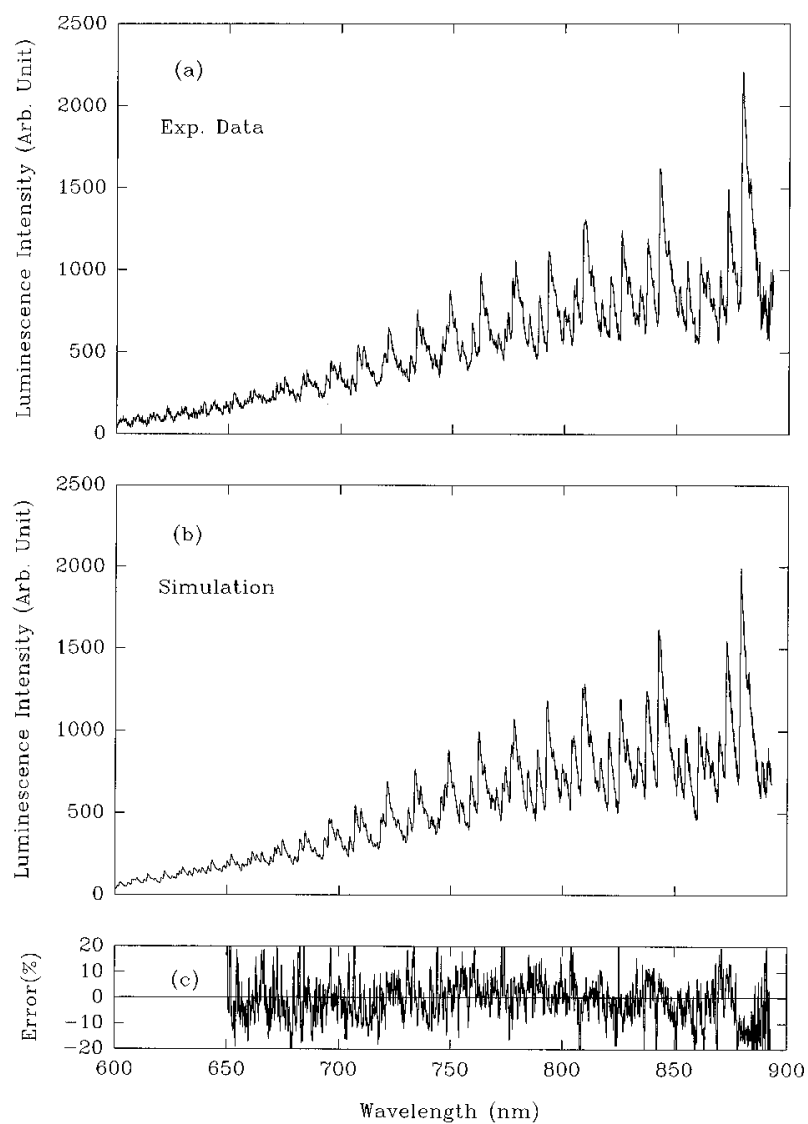

Figure 3. Detection efficiency corrected chemiluminescence spectra of chlorine atom recombination reactions at 4.0 mbar chlorine pressure and $300 \mathrm{~K}$ temperature. (a and b) Experimental and simulated spectra, respectively. (c) Percentage deviations between those of (a) and (b). The luminescence intensity is in proportion to the photon number, and the vacuum wavelengths are used in the abscissa coordinate.

distribution with $T=400 \mathrm{~K}$ for the low vibrational states, and with the population enhancement factors of 1.4, 2.2, 3.0, and 4.0 to the corresponding Boltzmann population distribution for the vibrational states $4,5,6$, and 7 , respectively. The populations of the remaining higher vibrational states were too low to be determined in the present experiments. The fitted vibrational population of the $A$ state is in $330 \mathrm{~K}$ Boltzmann distribution up to the vibrational quantum number of 2 . The intensities of the remaining higher vibrational states were too weak to be determined precisely. The rotational temperatures of both states were set at the experimental temperature of $300 \mathrm{~K}$. The ratio of the total photon emission between the $A$ and $B$ states in the 650$893 \mathrm{~nm}$ range is 0.32 . Apparently, the steady-state photon emission of the $A$ state in the chlorine atom recombination reactions is not negligible for any quantitative study of the reactions.

Knowing the relative luminescence weight 0.50 , and also the ratio of the $A \rightarrow X$ to $B \rightarrow X$ transition dipole moment $1 / 4.8,{ }^{17}$ the ratio of the $A$ to $B$ state population could be determined to be 12 under the present steady-state recombination condition. We shall come back to this point in the following reaction mechanism subsection.

Analysis of the Chemiluminescence Spectra in the HighPressure Regime. When the argon pressure is higher than 1 bar, spectral frequency shifts and line broadening become significant. Under the binary impact approximation, the line shapes of the electronic transitions follow the Lorentzian distribution: ${ }^{24}$

$$
I(\omega)=\frac{\gamma}{2 \pi} \frac{1}{\left(\omega-\omega_{0}-\Delta\right)^{2}+(\gamma / 2)^{2}}
$$
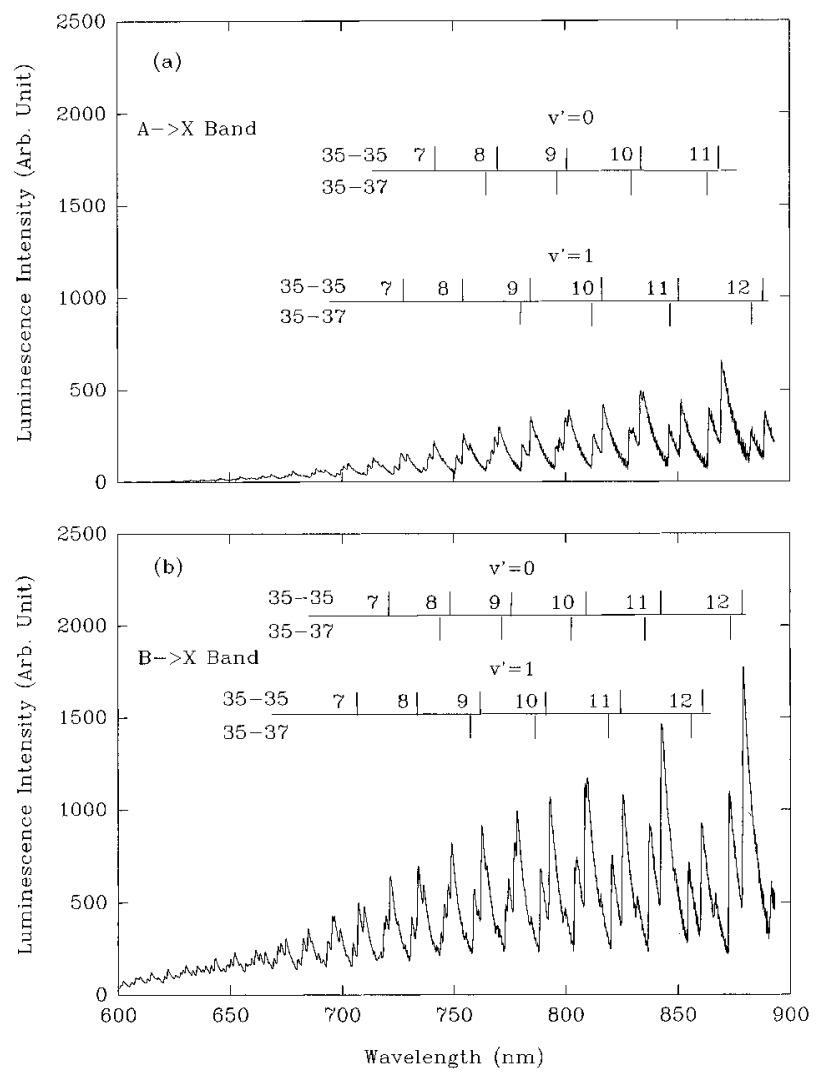

Figure 4. Luminescence spectrum of Figure 3 was decomposed into the $A \rightarrow X$ and $B \rightarrow X$ emissions. The total intensity ratio between these two transitions over the wavelength range $650-890 \mathrm{~nm}$ is 0.32 . The major vibrational bands for the two main isotopic chlorine molecules are also indicated.

in which $\omega$ is the frequency, $\omega_{0}$ is the unperturbed frequency, $\Delta$ is the shift of the frequency, and $\gamma$ is the width of the distribution. In the following simulation the rovibronic line shapes of the chlorine molecules were assumed to follow the same distribution, and $\Delta$ and $\gamma$ are two independent adjustable parameters in the calculations of the theoretical spectra.

The Franck-Condon factors were assumed to be identical with the free state values. The rotational temperature was set at the experimental temperature of $300 \mathrm{~K}$. The relative luminescence weights between the $A$ and $B$ states, the values of the pressure frequency shifts and broadenings, and the vibrational temperatures were then obtained by the same procedure as described in the previous subsection over the pressure range 1-170 bar. Since the peak positions of the vibrational bands are mainly determined by the frequency shifts, and the band widths were mainly determined by the frequency broadenings, these two adjustable parameters could be determined independently in the fitting procedure. In contrast to the very low pressure cases, in which there were still some residual non-Boltzmann population distribution in the higher vibrational states, it was found that as long as the argon pressures were higher than about 64 mbar, the vibrational population distribution could be adequately represented by a single temperature and also that the vibrational temperatures of the $A$ and $B$ states are actually quite close to each other. A single vibrational temperature was employed to simulate the observed spectra.

Figure 5 shows the full chlorine emission spectra at 30 and 170 bar argon pressures, respectively, and their corresponding simulated spectra. As shown in the enlarged portion of the figure, the agreement between the experimental and theoretical spectra is excellent down to the detailed spectral features. The 

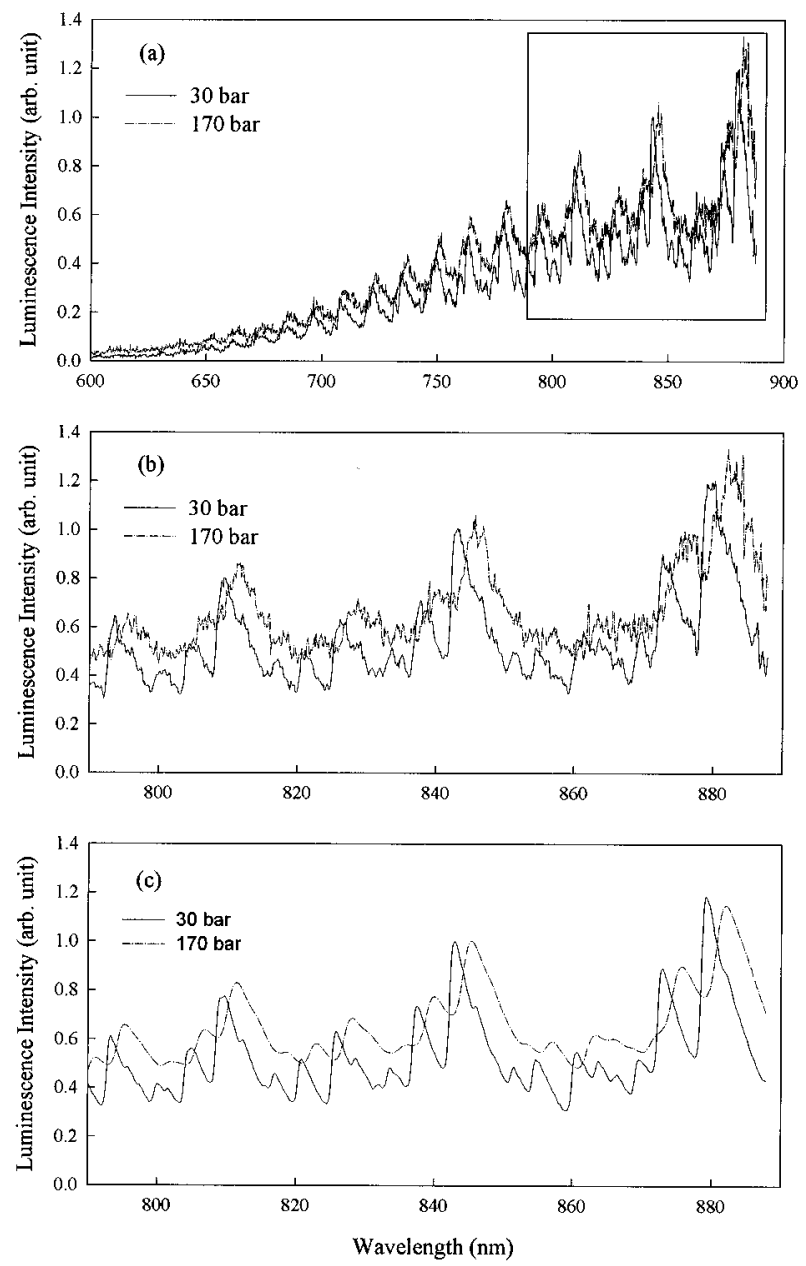

Figure 5. Luminescence spectra taken at 30 and 170 bar argon pressure. (a and b) Experimental spectra. (c) Corresponding simulated spectra. The sharp spikes in the experimental spectra were due to the defects of some picture cells of the ICCD.

TABLE 1: Fitted Parameters of the Simulated Emission Spectra at 5.33 mbar $\mathrm{Cl}_{2}{ }^{a}$

\begin{tabular}{clccc}
\hline$P_{\mathrm{Ar}}($ bar $)$ & weight $(A / B)$ & shift $\left(\mathrm{cm}^{-1}\right)^{b}$ & broadening $\left(\mathrm{cm}^{-1}\right)$ & $T(\mathrm{vib})$ \\
\hline $0.064^{c}$ & 0.50 & 0 & 0 & 340 \\
1.00 & 0.46 & 0 & 0 & 325 \\
3.00 & 0.41 & 0 & 1 & 331 \\
6.00 & 0.43 & 0 & 3 & 310 \\
10.0 & 0.44 & -1 & 3 & 326 \\
15.0 & 0.49 & -2 & 3 & 327 \\
20.0 & 0.5 & -3 & 4 & 328 \\
25.0 & 0.47 & -2 & 5 & 328 \\
30.0 & $0.46 \pm 0.01$ & $-2 \pm 2.2$ & $6 \pm 0.5$ & 325 \\
40.0 & $0.51 \pm 0.01$ & $-2.5 \pm 2.1$ & $8 \pm 1$ & 320 \\
50.0 & $0.49 \pm 0.02$ & $-4 \pm 2.2$ & $8 \pm 1$ & 319 \\
75.0 & $0.51 \pm 0.02$ & $-12 \pm 2.2$ & $13 \pm 1$ & 321 \\
100 & $0.47 \pm 0.01$ & $-14 \pm 2.2$ & $17 \pm 1$ & 337 \\
150 & $0.47 \pm 0.02$ & $-23 \pm 2.2$ & $25 \pm 1$ & 374 \\
170 & $0.43 \pm 0.01$ & $-26.5 \pm 3.2$ & $27 \pm 1$ & 344
\end{tabular}

${ }^{a}$ Neat $\mathrm{Cl}_{2}$ (4.0 mbar) sample: weight $(A / B)=0.5 ; T_{B \text { state }}(\mathrm{vib})=$ $400 \mathrm{~K} ; T_{\text {Astate }}(\mathrm{vib})=330 \mathrm{~K} ; T(\mathrm{rot})=300 \mathrm{~K}$, see the text for the details. ${ }^{b}$ The negative values indicate a red shift. ${ }^{c}$ For this argon pressure, $\mathrm{Cl}_{2} 2.67$ mbar.

regular sharp spikes in the experimental spectra were due to the defects of some picture cells of the ICCD. Table 1 lists the relative luminescence weights of the $A$ and $B$ states, the frequency shifts and line broadenings, and the vibrational temperatures over $0.064-170$ bar argon pressure. The pressuredependent trends of the frequency shifts, line broadening, and relative luminescence weights are also shown in Figure 6. In
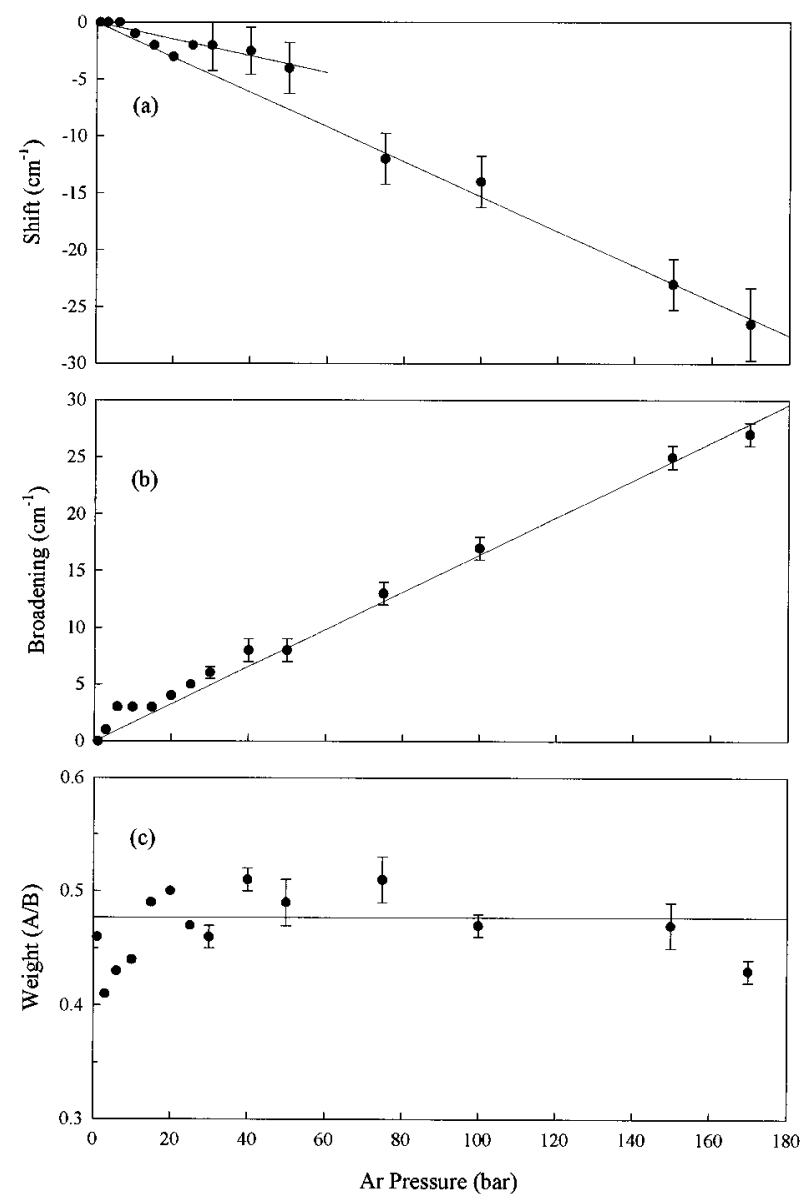

Figure 6. Pressure frequency shifts, line broadenings, and relative luminescence weights of the $A$ and $B$ states as a function of the argon pressure. See the text for the details of the fitted solid lines.

Figure 6a, the two solid lines represent the least-squares fittings of the frequency shifts as a linear function of the pressure in the high pressure (75-170 bar) and the low pressure (25-50 bar) regimes, respectively. Due to the uncertainties in the broadening determination, those data points below 20 bar were not used in the fitting procedure. Apparently, two different frequency-shift variations are operated separately in the lowand high-pressure domains. On the other hand, for the line broadenings, the linear least-squares fitting of the high-pressure line broadenings shown in Figure $6 \mathrm{~b}$ suggests that the linear relation is adequate in representing the present full range of the experimental results.

The mean relative $A / B$ luminescence weight over the studied pressure range is 0.47 and is shown in Figure $6 \mathrm{c}$ by the solid horizontal line. The vibrational temperatures are essentially equal to $330 \mathrm{~K}$. As mentioned in the previous subsection, in the low-pressure regime, such as under neat 4.0 mbar chlorine condition, the $A / B$ relative weight was determined to be 0.50 . One could conclude that the relative weights are a constant over the whole pressures measured in this study. However, the vibrational temperature increases to $400 \mathrm{~K}$ at the neat $4.0 \mathrm{mbar}$ chlorine pressure. Apparently, the argon buffer gas does not affect preferentially either the $A$ or $B$ state in terms of the recombination reaction mechanism and the electronic quenching. It does thermalize the extra vibrational energies for both states.

The other possible emission $A^{\prime} \rightarrow X$, which was observed in the matrix-isolated systems, ${ }^{11,12}$ was also looked for in both the detection time and the emitting wavelength domains. The emission was not observed up to 170 bar of argon pressure and over the observation time window up to $200 \mathrm{~ms}$ after the laser 
shots. The quantitative agreement between the simulated and experimental spectra also excluded the possible emission of this transition.

Mechanism of the Radiative Recombination Reactions of the Chlorine Atoms. In the low-pressure regime, the radiative recombination reactions of the chlorine atoms have traditionally been attributed to the recombination to the $B$ state. Along this line, the recombination reaction rate into the $B$ state was determined to be $5.0 \times 10^{-34} \mathrm{~cm}^{6}$ molecule ${ }^{-2} \mathrm{~s}^{-1}$ at $298 \mathrm{~K}$ by Clyne and Smith, ${ }^{8}$ a value corresponding to a recombination quantum efficiency of 0.043 . The recombination mechanism has been rationalized as an inverse predissociation process of the recombining $\mathrm{Cl}\left({ }^{2} P_{3 / 2}\right)+\mathrm{Cl}\left({ }^{2} P_{3 / 2}\right)$ atoms. ${ }^{8}$ Although it has been noticed that under steady-state conditions, the vibrational population of the $B$ state observed in the afterglow experiment could not be quantitatively explained by the above simplified mechanism. Collisional population transfer from some highly vibrationally excited states of the $X$ state was proposed to reconcile the discrepancy in the $B$ state vibrational population. ${ }^{8}$ In the following discussion, we shall reassess these earlier rationalizations.

The present low-pressure results suggest that $\mathrm{A} / B$ population ratio is 12 under the steady-state condition. For the highpressure measurements, assuming that the Franck-Condon factors and the transition dipole moments were equal to those of the free state, one also obtained the same $A$ to $B$ population ratio as that in the low pressure regime, as suggested by the constancy of the $A / B$ luminescence weights. Apparently, the argon buffer gas exerts the same effect on the rates of the recombination and the electronic quenching processes for the $A$ and $B$ states. Since in the neat low-pressure chlorine samples, the third body for the recombination reactions is the chlorine molecules, while under the high-pressure conditions the third body is Ar, and the recombination rate constant in the chlorine molecules is $5.5 \times 10^{-32}$ and $1.21 \times 10^{-32} \mathrm{~cm}^{6}$ molecule ${ }^{-2}$ $\mathrm{s}^{-1}$ in argon at $298 \mathrm{~K}$, one plausible explanation is that either the relative recombination efficiency between the A and B state is independent of the third bodies and the electronic quenching rate constants of the $\mathrm{A}$ and $\mathrm{B}$ states are similar to each other or that the interelectronic state transfer between the two states are faster than those of the electronic quenching and radiative processes under the present experimental conditions. Note that the second condition alone is enough to ensure that the $A$ and $B$ states are indistinguishable under the present resolution of the observation time and therefore manifest a constant relative weight. It is known that the energy difference between the $B$ and $A$ states is $365 \mathrm{~cm}^{-1}$. Both states have similar fundamental vibrational frequencies. If these states were in thermal equilibrium at $300 \mathrm{~K}$ and if one takes into account their electronic degeneracies, one could obtain the population ratio of $A$ to $B$ being 11.5. This value is in good agreement with the present experimental population ratio of 12. This observation supports that the $B$ and $A$ states are essentially in thermal equilibrium distribution except under the very low pressure condition. This implies that the interelectronic exchange rates and the vibrational relaxation rates are much faster than those of the radiative and electronic quenching processes for the $A$ and $B$ states. An additional note is that the fast interelectronic exchange condition does not exclude the possibility of the constancy of the relative recombination efficiencies between the $A$ and $B$ states under the present experimental conditions.

With information on the steady-state recombination populations, the electronic quenching and vibrational-redistribution rate constants, ${ }^{25,26}$ and also the transient recombination experiments, ${ }^{27}$ one could find that the following traditional radiative recombination mechanism, which is only involved in the $B$ state, is too simplified to be able to account for the experimental observations:

$$
\begin{array}{cc}
\mathrm{Cl}+\mathrm{Cl}+\mathrm{M} \rightarrow \mathrm{Cl}_{2}(B, v)+\mathrm{M} \quad \text { recombination } \\
\mathrm{Cl}_{2}(B, v)+\mathrm{M} \leftrightarrow \mathrm{Cl}_{2}(B, v+\Delta v)+\underset{\mathrm{M}}{\text { vibrational relaxation }} \\
\mathrm{Cl}_{2}(B, v)+\mathrm{M} \rightarrow \mathrm{Cl}_{2}(N)+\mathrm{M} \quad \text { electronic quenching } \\
\mathrm{Cl}_{2}(B, v) \rightarrow \mathrm{Cl}_{2}(X)+h v \quad \text { photon emission }
\end{array}
$$

in which the $B$ state was further partitioned by vibrational quantum number $v$, the symbol $N$ just indicates the nonradiative electronic states, $\Delta v$ is any vibrational jump within the allowed vibrational states, and $\mathrm{M}$ represents the third body. Incorporation of the interelectronic coupling to a neighboring electronic state into the above single electronic state mechanism is required to account for the available experimental observations. It has been known that the $A$ and $B$ states of the free chlorine molecules are coupled together through the heterogeneous perturbation interaction. ${ }^{11,28}$ It is also known that for homonuclear diatomic molecules, such as $\mathrm{N}_{2}$, the collisional population transfer within the same spin state is quite efficient if the energy difference between them is within the collisional energy. ${ }^{29}$ On the basis of the results of the present study, a natural extension of the above mechanism is the introduction of collisional interelectronic state exchange between $B(v)$ and $A(\mu)$ if their energy difference is within the thermal energy $k T$. In addition to the regular recombination, vibrational relaxation, and quenching processes within the $A$ state, the additional elementary step would be

$$
\mathrm{Cl}_{2}(B, v)+\mathrm{M} \leftrightarrow \mathrm{Cl}_{2}(A, \mu)+\mathrm{M}
$$

in which $v$ and $\mu$ are the vibrational quantum number.

On the baiss of the above mechanism, one could model the steady-state experiment of Clyne et al. and derive the corresponding apparent third-order rate constant for formation of both the $B$ and $A$ states, instead of the traditional pure $B$ state. ${ }^{8}$ One would find that for the earlier measured recombination rate constant of the $B$ state to be held, the recombination rate constant of the $A$ state should be much less than that of the $B$ state and also the product of the $A$ state radiative rate constant and the $B$ state quenching constant should be much less than that of the $B$ state radiative rate constant and the $A$ state quenching constant. However, available theoretical arguments suggest that the $A$ state recombination rate constant is expected to be larger than that of the $B$ state. ${ }^{30}$ Taking this constraint into account, one would find that the measured recombination rate constant of the $B$ state by Clyne et al. is actually just its upper bound value.

For a better understanding of the kinetics of the atomic chlorine recombination reactions, a detailed knowledge of vibrational state to state redistribution rate constants and the interelectronic collisional transfer constants is needed. This information is usually not easy to obtain. Nevertheless, by employing the monoexponential, energy-independent model for the vibrational redistribution rate constants ${ }^{25}$ and assuming an average rate constant for collisional population transfer if the energy is within the thermal energy, one could solve the above coupled master equations of the $A$ and $B$ states. A set of the rate constants for the above mechanism which yielded a selfconsistent solutions within the available experimental observations could be obtained in the low-pressure regime. A detailed 
analysis of the master equations along with the results of transient recombination measurements shall be reported elsewhere. ${ }^{27}$

Pressure Line Broadening and Frequency Shifts of the Chlorine Molecule Emissions. Theories of pressure line broadening and frequency shift for electronic transitions in the gas phase are generally based on either the binary impact model or the statistical model. ${ }^{24,31}$ It has been established that the former model is applicable to the lower pressure regime and the latter model is suitable for high-pressure systems. The exact applicable pressure range of the impact model is determined by the interaction strength between the chromophore and the bath gas and by the temperature. ${ }^{24}$ Since the experimental pressure line broadenings and frequency shifts are directly proportional to the bath pressure and also because the interaction between the chlorine molecules and argon is expected to be of the weak van der Waals type, the impact model was employed to estimate the average potential energy difference between the emitting energy states of the $\mathrm{Cl}_{2} / \mathrm{Ar}$ system. Neglecting anisotropy and assuming that the potential difference between the $B$ and $X$ state caused by the collision of argon is in the Lennard-Jones potential form:

$$
\Delta V(r)=C_{12} r^{-12}-C_{6} r^{-6}
$$

in which $r$ is the distance between the center-of-mass of chlorine molecule and argon, one may follow the well-developed procedure for the calculations of the pressure line broadening and frequency shift ${ }^{32}$ and obtain an average $C_{6}=(1.1 \pm 0.4)$ $\times 10^{-60} \mathrm{erg} \mathrm{cm}^{6}$ and $C_{12}=(0.8 \pm 0.6) \times 10^{-104} \mathrm{erg} \mathrm{cm}^{12}$ for the present system over the argon pressure $25-50$ bar. In these calculations, since the equilibrium bond distance of the $X$ state $1.987 \AA$ is much shorter than that of the $B$ state $2.435 \AA$, the sign of $C_{12}$ has been assumed to be positive. The value of $C_{6}$ was first determined through the ratio of the observed frequency shifts and line broadenings. The value of $C_{12}$ is then proportional to the 2.2 power of the absolute value of $C_{6}$ and can be obtained accordingly. ${ }^{32}$ The uncertainty of the $C_{12}$ value is inherent much larger than that of $C_{6}$ calculated by this method. Compared with the $\mathrm{Ar}-\mathrm{Ar}$ Lennard-Jones parameters $C_{6}=1.04$

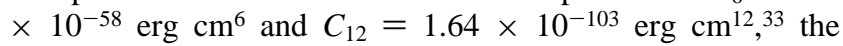
obtained potential difference is much smaller than that of the $\mathrm{Ar}-\mathrm{Ar}$ van der Waals molecule. It suggests that the interaction strength between the $B$ state of the chlorine molecule and the argon atom is quite similar to that in the case of the $X$ state. For $\mathrm{ArCl}_{2}$, it was known that the bond distance from the argon to the chlorine center of mass is $3.7 \AA$ for both the $X$ and $B$ electronic states of the complex and that their corresponding bond energies are 188 and $178 \mathrm{~cm}^{-1}$, respectively. ${ }^{34}$ The similarity of the bond energies and equilibrium bond distances between these two electronic states of the $\mathrm{ArCl}_{2}$ complex is consistent with the present conclusion about the similarity of the interaction strengths for both the $X$ and $B$ states of $\mathrm{Cl}_{2}$ in collision with argon.

\section{Conclusions}

The present chemiluminescence spectral analysis of the radiative chlorine atom recombination reactions suggests that the luminescence contribution of the $A \rightarrow X$ transition is important for the total luminescence spectra of the atomic chlorine recombination reactions. The constancy of the relative $A / B$ luminescence weights over the wide range of the pressures suggests that the traditional reaction mechanism of the radiative chlorine atom recombination was oversimplified. The population ratio of the $A$ to $B$ state under the present experimental conditions indicated that the $A$ and $B$ states are essentially in the Boltzmann population distribution of the room temperature and that the rate of the interelectronic population exchange is much faster than those of the electronic quenching and photonemission processes. The average isotropic potential difference between the emitting and the ground state of the chlorine molecules during the collision of the argon atoms was approximated by the Lennard-Jones potential form and, with the help of the measured pressure line broadening and frequency shift data, was determined by the binary impact model.

Acknowledgment. The financial support of this work by the National Science Council, Republic of China, is gratefully acknowledged.

\section{References and Notes}

(1) Bader, L. W.; Ogryzlo, E. A. J. Chem. Phys. 1964, 41, 2926.

(2) Hutton, E.; Wright, M. Trans. Faraday Soc. 1965, 61, 78. 424.

(3) Clyne, M. A. A.; Coxon, J. A. Proc. R. Soc. London A 1967, 298,

(4) Clyne, M. A. A.; Stedman, D. H. Trans. Faraday Soc. 1968, 64, 1816.

(5) Browne, R. J.; Ogryzlo, E. A. J. Chem. Phys. 1970, 52, 5774.

(6) Coxon, J. A. In Molecular Spectroscopy; Chem. Soc. 1973, 1, 177.

(7) Coxon, J. A.; Shanker, R. J. Mol. Spectrosc. 1978, 69, 109.

(8) Clyne, M. A. A.; Smith, D. J. J. Chem. Soc., Faraday Trans. 2, 1979, 75,704

(9) Kernashitskii, L. A.; Nosenko, V. E.; Naumov, V. V.; Kochelap, V. A.; Izmailov, I. A. Chem. Phys. Lett. 1985, 116, 197.

(10) Prigent, P; Giraud, X.; Brunet, H. Proc. SPIE-Int. Soc. Opt. Eng. 1989, 1031, 408.

(11) Bondybey, V. E.; Fletcher, C. J. Chem. Phys. 1976, 64, 3615.

(12) McCaffrey, J. G.; Kunz, H.; Schwentner, N. J. Chem. Phys. 1992, 96, 155.

(13) Hwang, C.-J.; Jiang, R.-C.; Su, T.-M. J. Chem Phys. 1986, 84, 5095.

(14) Weng, C.-J.; Ho, T.-I.; Su, T.-M. J. Phys. Chem. 1987, 91, 5235.

(15) Hwang, M.-D.; Jiang, R.-C.; Su, T.-M. J. Chem Phys. 1989, 91, 1626.

(16) Song, T.-T.; Su, T.-M. J. Phys. Chem., following paper in this issue. (17) Ishiwata, T.; Ishiguro, A.; Obi, K. J. Mol. Spectrosc. 1991, 147 , 300. 193.

(18) Gibson, G. E.; Rice, O. K.; Bayliss, N. S. Phys. Rev. 1933, 44,

(19) Coxon, J. A. J. Mol. Spectrosc. 1980, 82, 264.

(20) Douglas, A. E.; Hoy, A. R. Can. J. Phys. 1975, 53, 1965.

(21) Tellinghuisen, P. C.; Guo, B.; Chakraborty, D. K.; Tellinghuisen, J. J. Mol. Spectrosc. 1988, 128, 268.

(22) Ishiwata, T.; Kasai, Y.; Obi, K. J. Chem. Phys. 1991, 95, 60.

(23) Cooley, J. W. Math. Comput. 1961, 15, 363.

(24) Sobelman, I. I.; Vainshtein, L. A.; Yukov, E. A. Excitation of Atoms and Broadening of Spectral lines; Springer-Verlag: New York, 1981.

(25) Clyne, M. A. A.; Martinez, E. J. Chem. Soc. Faraday Trans. 2, 1980, 76, 1275.

(26) Troe, J. J. Chem. Phys. 1977, 66, 4745.

(27) Chang, L.-C.; Su, T.-M., unpublished results.

(28) Martinez, E.; Basterrechea, F. J.; Cabanas, B.; Puyuelo, P.; Lopez, M. R. Laser Chem. 1992, 12, 75.

(29) Bachmann, R.; Li, X.; Ottinger, Ch.; Vilesov, A. F.; Wulfmeyer, V. J. Chem Phys. 1993, 98, 8606.

(30) Smith, I. W. M. Int. J. Chem. Kinet. 1984, 16, 423.

(31) Kalbfleisch, T.; Fan, R.; Roebber, J.; Moore, P.; Jacobsen, E.; Ziegler, L. D. J. Chem. Phys. 1995, 103, 7673.

(32) Hindmarsh, W. R.; Petford, A. D.; Smith, G. Proc. R. Soc. London A 1967, 297, 296.

(33) Maitland, G. C.; Rigby, M.; Smith, E. B.; Wakeham, W. A. Intermolecular Forces, Their Origin and Determination; Oxford: New York, 1981.

(34) Evard, D. D.; Cline, J. I.; Janda, K. C. . J. Chem. Phys. 1988, 88, 5433.

JP960499C 